\title{
Nucleation threshold and deactivation mechanisms of nanoscopic cavitation nuclei
}

\author{
Bram M. Borkent, Stephan Gekle, Andrea Prosperetti, ${ }^{a)}$ and Detlef Lohse ${ }^{\text {b) }}$ \\ Physics of Fluids, Faculty of Science and Technology and J.M. Burgers Centre for Fluid Dynamics, \\ Impact Institute and MESA ${ }^{+}$Institute for Nanotechnology, University of Twente, \\ P.O. Box 217, 7500 AE Enschede, The Netherlands
}

(Received 26 May 2009; accepted 11 September 2009; published online 19 October 2009)

\begin{abstract}
The acoustic nucleation threshold for bubbles trapped in cavities has theoretically been predicted within the crevice theory by Atchley and Prosperetti ["The crevice model of bubble nucleation," J. Acoust. Soc. Am. 86, 1065 (1989)]. Here, we determine this threshold experimentally, by applying a single pressure pulse to bubbles trapped in cylindrical nanoscopic pits ("artificial crevices") with radii down to $50 \mathrm{~nm}$. By decreasing the minimum pressure stepwise, we observe the threshold for which the bubbles start to nucleate. The experimental results are quantitatively in good agreement with the theoretical predictions of Atchley and Prosperetti. In addition, we provide the mechanism which explains the deactivation of cavitation nuclei: gas diffusion together with an aspherical bubble collapse. Finally, we present superhydrophobic nuclei which cannot be deactivated, unless with a high-speed liquid jet directed into the pit. () 2009 American Institute of Physics.
\end{abstract}

[doi:10.1063/1.3249602]

\section{INTRODUCTION}

Water can be ruptured at much smaller tensile stresses than theoretically expected. ${ }^{1}$ The reason for this discrepancy could be the existence of small inhomogeneities in the liquid, which may exist even when special care on the cleanliness of the water has been taken. ${ }^{2}$ The inhomogeneities, whatever their origin might be, have received the generic name "cavitation nuclei," while the bubble generation produced in this way is termed heterogeneous nucleation. Cavitation nuclei are generally long lived and it is believed that they consist at least in part of a volume of gas. ${ }^{3}$ This observation excludes the possibility of the inhomogeneities being free spherical gas bubbles, as these are unstable. To account for stable gaseous cavitation nuclei, two types are distinguished in literature: bubbles stabilized by a skin (see Ref. 3 and references therein) and bubbles trapped inside a surface defect ("crevice model"). ${ }^{4-9}$

The principle of the crevice model dates back to $1944^{4,10}$ and has found extensive qualitative experimental evidence over the years. Greenspan and Tschiegg, ${ }^{11}$ for example, reported that removing particles larger than $0.2 \mu \mathrm{m}$ in diameter increased the tensile strength of water to about 200 bar (see also Refs. 2 and 12). Others found that the addition of suspended particles lowers the nucleation threshold, ${ }^{13-18}$ while pre-experimental pressurization of water increases the nucleation threshold. ${ }^{5,19}$ Although these findings are in line with the general idea of the crevice model, none of the experiments could quantitatively verify the theoretical crevice model as developed by Atchley and Prosperetti ${ }^{8}$ in 1989. One of the reasons is that their predictions are valid for a single cavity of a well-defined shape, while in practice the

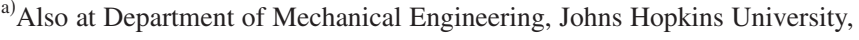
Baltimore, MD 21218, USA.

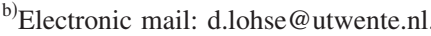

liquid usually contains a wide variety of nuclei of different sizes and shapes. Even in ultrapure water with a controlled number of microparticles, the sizes of the nuclei present on the microparticles can exhibit size variations yielding a wide range of thresholds. ${ }^{19}$

A step forward was achieved by Bremond et al..$^{20-22}$ who were able to create monodisperse cavitation nuclei by trapping gas inside cylindrical holes of well-defined shape etched in silicon surfaces using standard lithography techniques. Not only was the position and size of the nuclei perfectly controlled but the nucleation event itself was also highly reproducible so that it could be followed in time with stroboscopic methods without the need of expensive highspeed cameras.

Both conditions, the reproducibility of the experiment and the monodispersity of the nuclei present at fixed positions, are important ingredients of this paper. Here, we have downscaled the micropits of Bremond et al. two orders of magnitude so that it becomes possible to experimentally verify the theoretical predictions made in the framework of the crevice model. This is the first aim of this paper. Second, we explore the mechanisms leading to the deactivation of nuclei after a single nucleation event. In addition, we show that superhydrophobic cavitation nuclei can nucleate hundreds of times, without being deactivated. Our observations and interpretations have implications for an increased understanding of the behavior of cavitation nuclei down to length scales of a few tens of nm ("surface nanobubbles"). ${ }^{23}$

\section{BRIEF THEORETICAL DESCRIPTION}

A comprehensive description and development of the crevice model can be found in the paper of Atchley and Prosperetti ${ }^{8}$ with extensions to any axisymmetric geometry, including cylindrical cavities, given by Chappell and Paine. ${ }^{24}$ 


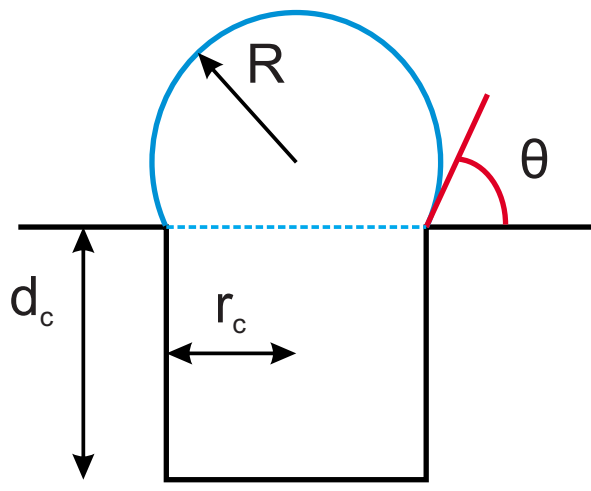

FIG. 1. (Color online) Cylindrical cavity with its dimensions. The initial gas-liquid interface is flat (dashed line), while the expanding bubble has a radius of curvature $R$ and contact angle $\theta$ with the flat surface.

In both papers, the authors predicted the various nucleation thresholds as function of crevice shape, gas tension, and receding contact angle. Atchley and Prosperetti argued that a true nucleation event must be the result of the loss of mechanical stability of the nucleus, and calculated the threshold for two situations: The first threshold denotes the pressure at which the nucleus inside a crevice begins its unstable growth, and is the lowest pressure value belonging to a bubble reaching either the critical radius of curvature $R_{c}$, or the receding radius of curvature $R_{R}$, i.e., the radius of curvature at which the receding contact angle $\theta_{R}$ is reached. The second threshold is equivalent in definition, but holds for the bubble growing outside of the crevice mouth. The lower value of the first and second nucleation thresholds is the one for which the bubble grows explosively out of the cavity. For the case of a bubble trapped in a cavity with volume $V_{c}$ with its interface at the crevice mouth, the (second) nucleation threshold is given by

$$
p_{L}+\frac{2 \sigma}{R}=p_{v}+\frac{V_{0} p_{g, 0}}{V_{c}+(\pi / 3) g(\theta) R^{3}},
$$

with $p_{L}$ as the liquid pressure, $p_{v}$ as the vapor pressure, $V_{0}$ as the initial volume of the gas, $p_{g, 0}$ as the initial gas pressure in the bubble, $\sigma$ as the liquid-gas surface tension, and $(\pi / 3) g(\theta) R^{3}$ as the volume of the spherical-cap-shaped bubble with radius $R$ as it expands above the cavity ${ }^{8}$ (see Fig. 1). Here, $g(\theta)=\left[2+\left(2+\sin ^{2} \theta\right) \cos \theta\right]$ is a geometric function depending on the contact angle $\theta$. The right-hand side of Eq. (1) represents the expanding forces caused by the vapor and gas pressure, $p_{v}+p_{g}$, respectively, while the left-hand side represents the collapsing forces due to the liquid pressure and surface tension, respectively. Equation (1) implies that for nucleation to occur, the expanding forces should exceed the collapsing forces [condition (1)]. Second, this condition should persist for increasing $R$, i.e., $d\left(p_{g}+p_{v}\right) / d R>d$ $\left(p_{L}+2 \sigma / R\right) / d R$ [condition (2)].

Let us now consider the case-which we will examine experimentally - of a cylindrical cavity with radius $r_{c}$ and depth $d_{c}$, under the assumption that the initial gas-liquid interface at the crevice mouth is approximately flat (i.e., we assume a negligible effect of the hydrostatic pressure and gas-saturated water) so that we can write $V_{0}=V_{c}=\pi r_{c}^{2} d_{c}$. For

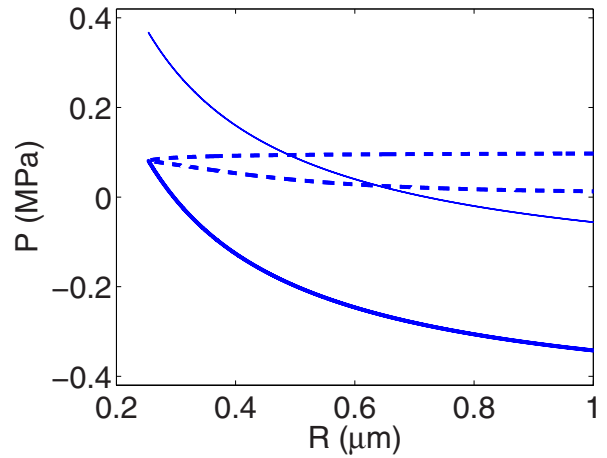

FIG. 2. (Color online) Graph of the expanding forces [right-hand side of Eq. (1), dashed line] and the collapsing forces [left-hand side of Eq. (1), solid lines] for a bubble expanding from a cylindrical pit with $r_{c}=250 \mathrm{~nm}$, $d_{c}=500 \mathrm{~nm}, p_{g, 0}=10^{5} \mathrm{~Pa}-p_{v}, p_{v}=73 \times 10^{2} \mathrm{~Pa}$, and $\theta_{R}=100^{\circ}$. The expanding forces (dashed line) show two branches corresponding to the possible solutions of $R$. The upper branch reflects the initially flat bubble during its first expansion phase: $R$ decreases from $R=R_{\infty}$ to $R=R_{R}$; the lower branch shows the solutions for the bubble expanding during its second phase: $R$ increases from $R=R_{R}$ to larger sizes. The collapsing forces are shown for two cases: (1) $p_{L}=-0.20 \mathrm{MPa}$ (thin solid line) and (2) $p_{L}=-0.486 \mathrm{MPa}$ (thick solid line). For case 1, the expanding forces dominate over the collapsing forces and the bubble will expand, until at $R=0.49 \mu \mathrm{m}$ a stable equilibrium is reached. No nucleation will occur in this case. In case 2 the expanding forces are larger than the collapsing forces for all possible solutions of $R$, and as a result the bubble will grow explosively.

$\theta_{R} \geq \pi / 2$ the nucleation threshold is the pressure needed to pull the bubble beyond its minimum radius while it expands from the cavity, i.e., $R_{\min }=R_{R}=r_{c} / \sin \theta_{R}$. Now, Eq. (1) can be rewritten as

$$
p_{L}=p_{v}+\frac{3 p_{g, 0}}{3+\left(r_{c} / d_{c}\right) g\left(\theta_{R}\right) / \sin ^{3} \theta_{R}}-\frac{2 \sigma \sin \theta_{R}}{r_{c}} .
$$

This prediction will be verified experimentally in this paper. At the point $R=R_{\min }=R_{R}$ the bubble experiences the maximum collapsing force. Any further reduction in the liquid pressure will make the bubble expand, thus reducing the surface tension pressure, and the bubble will grow explosively with the contact angle fixed at $\theta_{R}$. Equations (1) and (2) are illustrated in Fig. 2, for an air bubble trapped in a cylindrical pit with dimensions $r_{c}=250 \mathrm{~nm}$ and $d_{c}=500 \mathrm{~nm}$ and with $p_{g, 0}=10^{5} \mathrm{~Pa}-p_{v}, p_{v}=73 \times 10^{2} \mathrm{~Pa}$, and $\theta_{R}=100^{\circ}$. The graph shows the expanding and the collapsing forces for two cases: (1) $p_{L}=-0.20 \mathrm{MPa}$ (Blake threshold $^{25,26}$ for a free bubble with $R_{0}=r_{c}$ ) and (2) $p_{L}=-0.486 \mathrm{MPa}$ [prediction of Eq. (2)]. From this plot it is readily seen that the expanding pressure in the second case is always larger than the collapsing pressure, and that $d\left(p_{g}+p_{v}\right) / d R>d\left(p_{L}+2 \sigma / R\right) / d R$.

For $\theta_{R}<\pi / 2$ the nucleation threshold is much more complicated to calculate. At $R=R_{\min }=r_{c}$ the collapsing force due to surface tension is indeed maximum, but now $d\left(p_{g}+p_{v}\right) / d R<d\left(p_{L}+2 \sigma / R\right) / d R$, and therefore $p_{L}\left(R_{\text {min }}\right)$ cannot be the nucleation threshold. Also $p_{L}\left(R_{R}\right)$ is not the correct threshold, as this gives a stable equilibrium in the upper branch of the compressive force curve. Instead, the threshold needs to be found by numerically solving 


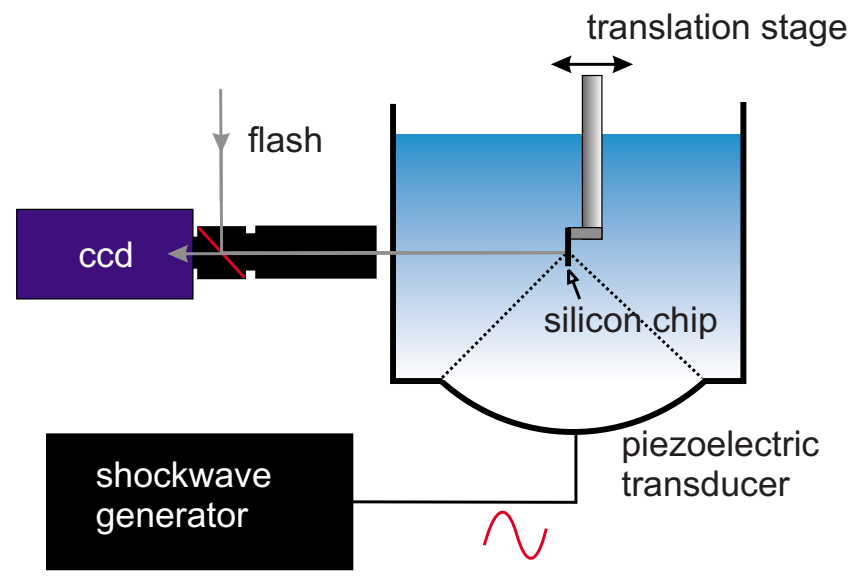

FIG. 3. (Color online) Sketch of the experimental setup.

$d\left(p_{g}+p_{v}\right) / d R=d\left(p_{L}+2 \sigma / R\right) / d R$, which is an implicit equation as the contact angle depends on the radius $R$, through $\sin \theta=r_{c} / R$, as long as the receding contact angle has not yet been reached. In the prediction of $p_{L}$ the gas term is significant for cavities down to a few hundreds of nanometers in radius and will therefore be taken into account in the present analysis.

\section{MATERIALS AND METHODS}

\section{A. Experimental setup}

The experimental setup to investigate the nucleation behavior of bubbles trapped in well-defined cavities is similar to that used by Bremond et al. ${ }^{20-22}$ and sketched in Fig. 3. Cavitation is induced by a focused shock wave generator (Piezoson 100, Richard Wolf GmbH) consisting of piezoelectric elements mounted on a spherical cap at the bottom of the liquid bath, which is filled with 11 of air-saturated water (Milli-Q Synthesis A10, Millipore). The cavitation activity is recorded optically with a charge coupled device camera (Flowmaster, LaVision) through a long-distance microscope (Model K2, Infinity). Illumination is provided by a flash lamp in reflection mode. The liquid pressure $p_{L}$ is obtained with the help of a calibrated glass fiber hydrophone (FOPH 500, RP Acoustics). The pressure is derived by measuring the reflected intensity of the laser beam at the fiber tip, which depends on the density of the water as affected by the local pressure. ${ }^{27}$ At the acoustic focus the pressure signal is typically characterized by a pressure peak (duration $\sim 1 \mu \mathrm{s}$ ) followed by a negative pressure phase $(\sim 5 \mu \mathrm{s})$. The intensity of the pressure pulse can be varied in 20 discrete steps. Since the smallest possible pressure decrease at the acoustic focus $\min \left(p_{L}\right)=-3.2 \mathrm{MPa}$ is already too large for our purpose, the samples are translated horizontally (away from the acoustic focus) along the line of sight, until the pressure signal is sufficiently weak that nucleation does not occur at the smallest pressure drop, but only at larger pressure decreases. The hydrophone tip (diameter: $100 \mu \mathrm{m}$ ) is positioned $\sim 0.1 \mathrm{~mm}$ in front of the chip surface, at an angle with the vertical plane of $10^{\circ}-20^{\circ}$. We took care of the close proximity between tip and surface by checking: (1) that the hydrophone tip end was not touching the chip surface (as this gave noisy

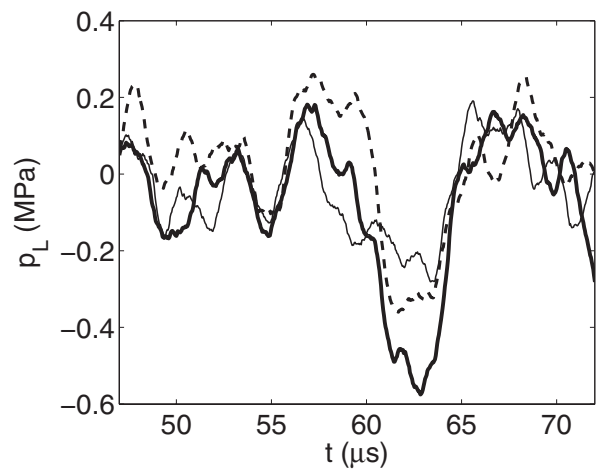

FIG. 4. Three pressure signals with increasing strength recorded at the chip surface $25 \mathrm{~mm}$ out of focus, corresponding to the experiment with sample B $\left(r_{c}=246 \mathrm{~nm}\right)$. Each line is the mean of 25 recordings. From these signals the minimum pressure can be extracted: $-0.24 \mathrm{MPa}$ (thin solid line), -0.35 $\mathrm{MPa}$ (dashed line), and $-0.54 \mathrm{MPa}$ (thick solid line).

data); (2) that the tip was in the optical focus of the camera (differences of $0.1 \mathrm{~mm}$ could be detected by comparing sharpness of the pictures). The corresponding pressure signals are recorded using a low pass filter (cutoff frequency 2.2 $\mathrm{MHz}$ ) and averaged over 25 recordings to reduce the noise. A typical recording of the averaged pressure signal obtained $25 \mathrm{~mm}$ out of focus is shown in Fig. 4 and corresponds to the experiment with the sample containing pits of $246 \mathrm{~nm}$ in radius.

\section{B. Samples with nanopits}

The substrates of interest are silicon pieces of $5 \times 5 \mathrm{~mm}^{2}$ diced from a $\mathrm{Si}(100)$ wafer. The nanoscopic cylindrical pits are directly etched into the substrate by a focused ion beam in a $6 \times 6$ square pattern, with $200 \mu \mathrm{m}$ distance between the pits. The resulting $1 \times 1 \mathrm{~mm}^{2}$ pattern is located at the center of the chip. In order to facilitate detailed imaging by atomic force microscopy (AFM) and scanning electron microscopy (SEM) identical pits are etched near the chip corner. Four samples (A)-(D) were studied with the following dimensions (determined with SEM) of the nanopits: (A) $r_{c}=495 \mathrm{~nm}$; (B) $r_{c}=246 \mathrm{~nm}$; (C) $r_{c}=53 \mathrm{~nm}$; (D) $r_{c}=50-60 \mathrm{~nm}$. In samples $\mathrm{A}-\mathrm{C}$ the pattern consisted of uniformly sized pits, with depth $d_{c}=2 r_{c}$, while in sample D each column of pits had different depths $(75,100,200,300,500$, and $1000 \mathrm{~nm}$ ), which influenced the radial pit size per column by a few nanometers $(50,50,50,55,57$, and $60 \mathrm{~nm}$, respectively); see Fig. 5 for the corresponding SEM pictures. Notice that $r_{c}$ has been measured precisely with SEM, while $d_{c}$ could not be measured and should therefore be regarded as an indicative value of the depth. In contrast to $r_{c}$, the exact depth of the pits (which only determines the initial gas volume) does not matter too much for the overall bubble dynamics, provided that the radius $r_{c}$ is small enough, which is the case in our experiments. After production the samples were cleaned ultrasonically in ethanol $(15 \mathrm{~min})$, followed by an oxygen plasma (5 min), a chemical cleaning step using a fresh (5:1) Piranha mixture (30 min), and again an ultrasonic bath in ethanol (15 min). This yielded clean and completely wetting substrates, which were characterized by a smoothly dewetting contact line (if not, the whole process was re- 


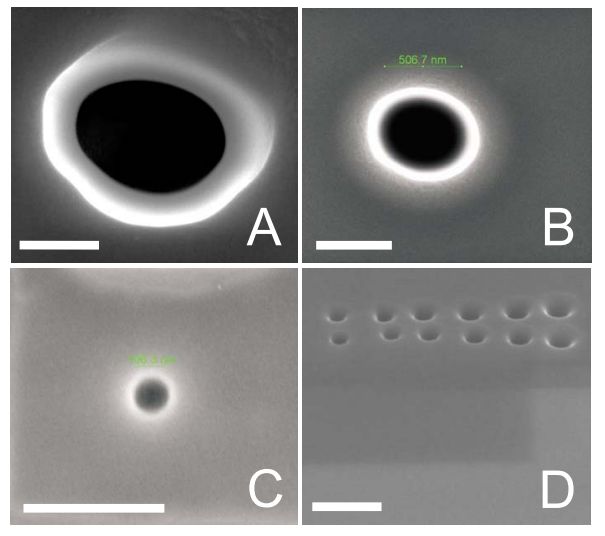

FIG. 5. (Color online) SEM images of samples A-D, respectively. The bars in each picture denote $500 \mathrm{~nm}$. The ellipsoidal shapes for samples A and B are due to drift in the SEM chamber, i.e., the pits have circular cross sections in reality.

peated). Subsequently, the samples were hydrophobized with $1-H, 1-H, 2-H, 2 H$-perfluorodecyltrichlorosilane following Ref. 28. The advancing and receding contact angles on the surface were $\theta_{a}=124^{\circ}$ and $\theta_{r}=100^{\circ}$. After immersion in water it was confirmed with AFM in tapping mode that a horizontal gas-liquid meniscus was present at the mouth of the pits.

\section{RESULTS}

\section{A. Nucleation from gas-filled nanopits}

To see whether it is possible to nucleate bubbles from nanopits as small as $53 \mathrm{~nm}$ in radius, samples $\mathrm{A}-\mathrm{C}$ were immersed in the liquid bath allowing air to be entrapped in the pits. In successive experiments the samples were placed at the acoustic focus of the shock wave generator and subjected to a pressure pulse with $p_{m}=\min \left(p_{L}\right)=-3.2 \mathrm{MPa}$. This value is sufficiently below the nucleation thresholds of the three samples, i.e., $-0.23,-0.48$, and $-2.59 \mathrm{MPa}$, respectively [see Eq. (2)], to expect nucleation of bubbles from the nanopits. The camera and flash were triggered a few microseconds after passage of the negative part of the shock wave to capture the expanding bubbles at maximum sizes. The result is depicted in Fig. 6 for samples A-C, respectively. Samples A and B showed almost perfect bubble patterns, with each bubble corresponding to the position of the nanoscopic cavitation nucleus. In each experiment, the cavitation nuclei had to be "reactivated" (filled with air again) since it was not possible to nucleate bubbles a second time

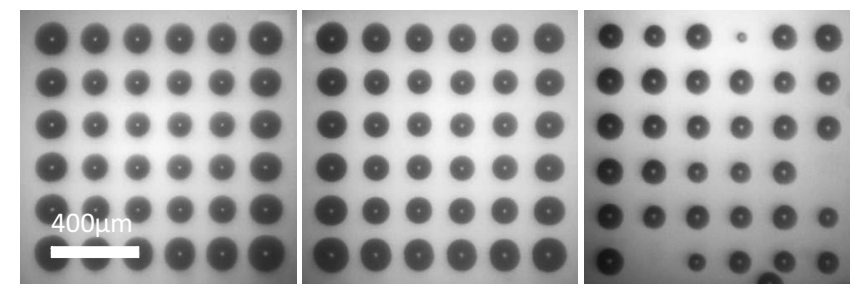

FIG. 6. Cavitation bubbles nucleated from cylindrical pits with radius $r_{c}=495 \mathrm{~nm}$ (left), $r_{c}=246 \mathrm{~nm}$ (middle), and $r_{c}=53 \mathrm{~nm}$ (right), and depth $d_{c}=2 r_{c}$ for a pressure pulse with $p_{m}=-3.2 \mathrm{MPa}$. without taking the sample out of the water first. ${ }^{21}$ With sample $\mathrm{C}$ a maximum amount of 34 bubbles could be nucleated in the first experiment, implying that it is indeed possible to nucleate bubbles from such small cavities. While samples A and B showed perfect reproducibility, the number of bubbles nucleating from sample $\mathrm{C}$ declined dramatically in later experiments, even when the negative pressure amplitude was increased to $-7 \mathrm{MPa}$. Presumably, small contaminant molecules had decreased the contact angle locally, leading to completely wetted nanopits. To test this possibility, the old hydrophobic coating was stripped off with an oxygen plasma and the sample was hydrophobized again through the cleaning and coating steps described before. This process indeed reactivated part of the nuclei ( $\sim 80 \%$ of the pits) although the number of bubbles declined again in successive experiments.

\section{B. Determination of the experimental nucleation threshold}

The experimental nucleation threshold of the nanosized cavities can be obtained by moving the samples to a position in the liquid bath where the pressure drop $p_{m}$ is sufficiently small that no nucleation occurs. By lowering $p_{m}$ stepwise the cavities will nucleate at a certain negative pressure amplitude, which is the experimental nucleation threshold. To observe the bubbles optically, it is not sufficient for the negative pressure to be low enough, but it should also last long enough in time. A lower limit to the time $\Delta t$ the bubble needs to grow to visible size is estimated by first estimating the critical size $R_{c, o}$ to be optically observable. We take $R_{c, o} \sim 3$ image pixels=3 pixels $\times 2.9 \mu \mathrm{m} /$ pixel $=8.7 \mu \mathrm{m}$. Now, using ${ }^{22,29} \dot{R}=\left\{(2 / 3)\left[\left(p_{v}-p_{m}\right) / \rho\right]\right\}^{1 / 2}$ with $\rho$ the liquid density and $\dot{R}$ the bubble wall velocity, it follows that $\Delta t=R_{c, o} / \dot{R}=0.9 \mu \mathrm{s}$ [sample (A)], $0.6 \mu$ s [sample (B)], and $\sim 0.3 \mu \mathrm{s}$ [samples (C) and (D)]. The minimum pressure level which lasts $\Delta t$ is the negative pressure amplitude $p_{m}$ of interest. Note that the difference with the absolute minimum pressure level is in most cases only a few percent, much smaller than the typical statistical error, which is $\sim 0.2 \mathrm{MPa}$ in the cases of samples $\mathrm{A}$ and $\mathrm{B}$, and $\sim 0.4-0.6 \mathrm{MPa}$ in the case of sample C.

Let us first consider the case of sample B $\left(r_{c}=246 \mathrm{~nm}, d_{c}=2 r_{c}\right)$. The sample was set $25 \mathrm{~mm}$ out of the acoustic focus, and three pressure pulses with increasing strength (Fig. 4) were applied successively, without taking the sample out of the water. A typical result is shown in Fig. 7. With the first pulse $\left(p_{m}=-0.24 \mathrm{MPa}\right)$ no cavitation bubbles could be observed [Fig. 7(a)]. The second pressure pulse $\left(p_{m}=-0.35 \mathrm{MPa}\right)$, resulted in a few nucleated bubbles (always in the top rows of the pattern), but the majority of the nuclei in the pattern still did not cavitate [Fig. 7(b)]. The third pressure pulse, with $p_{m}=-0.54 \mathrm{MPa}$, was able to nucleate all remaining nuclei [Fig. 7(c)]. Note that the nuclei which nucleated with the second pulse could not be nucleated with the stronger third pulse, implying that the nuclei can be used only once. Nuclei which were not nucleated during the second pulse, however, survived and were nucleated with the stronger third pulse. The experiment was re- 


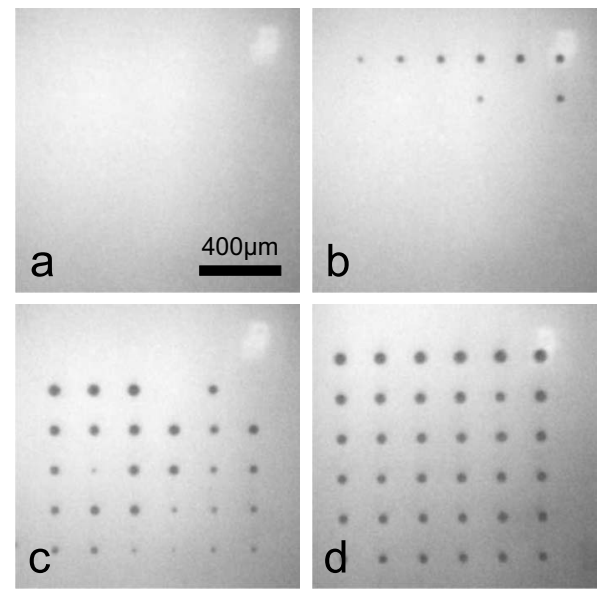

FIG. 7. Cavitation bubbles emerging from $6 \times 6$ cylindrical pits with $r_{c}=246 \mathrm{~nm}$ (sample B), for three successively applied pressure pulses: (a) $p_{m}=-0.24 \mathrm{MPa}$; (b) $p_{m}=-0.35 \mathrm{MPa}$; (c) $p_{m}=-0.54 \mathrm{MPa}$. (d) The full pattern develops when $p_{m}=-0.54 \mathrm{MPa}$ is applied without the other two preceding pulses.

peated eight times with reproducible results (Table I): on average 0,10 , and 26 bubbles were counted for the three applied pressure pulses, respectively. When the third pulse $\left(p_{m}=-0.54 \mathrm{MPa}\right)$ was applied without the other two preceding ones, the full pattern became visible [Fig. 7(d)]. Since the majority of the pits nucleated at $p_{m}=-0.54 \mathrm{MPa}$ it is reasonable to assume that this pressure amplitude is above the experimental nucleation threshold for sample $\mathrm{B}$, while the weaker pulse $\left(p_{m}=-0.35 \mathrm{MPa}\right)$ is (just) below the experimental nucleation threshold.

Since it was always the top row of pits plus some part of the second top row that nucleated at an apparently less negative $p_{m}$, it could very well be that there was a pressure variation along the chip surface in the vertical direction (i.e., direction of wave propagation) so that the most negative pressure occurred at the top row of the pits. In Figs. 7(c) and 7(d) we can indeed see that bubbles become slightly larger in the vertical direction, indicating a more negative pressure along this direction. With our setup we have not been able to measure a difference in pressure between top and bottom location of the pattern.

A similar experiment was carried out with sample A $\left(r_{c}=495 \mathrm{~nm}, d_{c}=2 r_{c}\right.$, Fig. 8). Again the sample was subjected to three successive pressure signals of decreasing negative pressure without being taken out of the water. For the lowest pressure amplitude $\left(p_{m}=-0.20 \mathrm{MPa}\right)$ no cavita-

TABLE I. Results of eight experiments with sample B $\left(r_{c}=246 \mathrm{~nm}, d_{c}\right.$ $=2 r_{c}$ ). In each experiment the minimum pressure $p_{m}$ is decreased in three successive steps. The majority of the pits nucleates at $p_{m}=-0.54 \mathrm{MPa}$.

\begin{tabular}{lccccccccc}
\hline \hline & \multicolumn{8}{c}{ Amount of bubbles } \\
\cline { 2 - 7 }$(\mathrm{MPa})$ & 1 & 2 & 3 & 4 & 5 & 6 & 7 & 8 & Mean \\
\hline-0.24 & 0 & 0 & 0 & 0 & 0 & 0 & 0 & 0 & 0 \\
-0.35 & 11 & 9 & 8 & 15 & 10 & 8 & 8 & 7 & 10 \\
-0.54 & 23 & 27 & 28 & 19 & 25 & 27 & 27 & 29 & 26 \\
\hline \hline
\end{tabular}

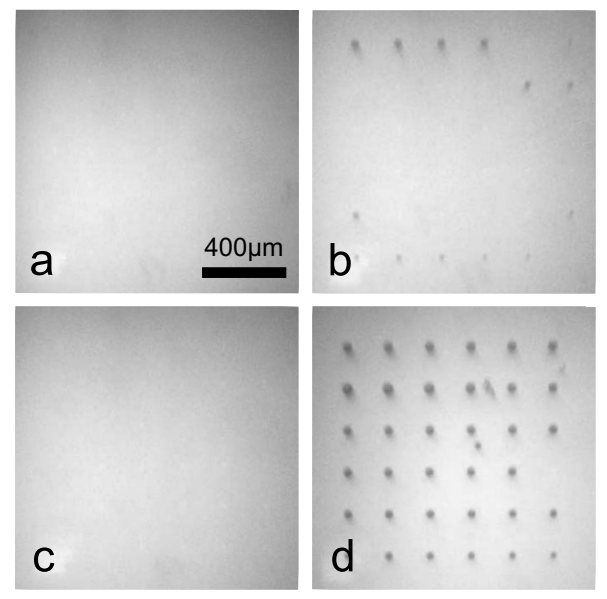

FIG. 8. Cavitation bubbles emerging from $6 \times 6$ cylindrical pits with $r_{c}=495 \mathrm{~nm}$ (sample A), for three successively applied pressure pulses: (a) $p_{m}=-0.20 \mathrm{MPa}$; (b) $p_{m}=-0.23 \mathrm{MPa}$; (c) $p_{m}=-0.34 \mathrm{MPa}$. (d) The full pattern develops when $p_{m}=-0.34 \mathrm{MPa}$ is applied without the other two preceding pulses.

tion bubbles could be detected optically [Fig. 8(a)]. A larger amplitude of $p_{m}=-0.23 \mathrm{MPa}$ yielded 14 bubbles of different sizes, with some of them barely visible [Fig. 8(b)], while a further reduction in the liquid pressure $\left(p_{m}=-0.34 \mathrm{MPa}\right)$ resulted in no visible bubbles at all [Fig. 8(c)]. What happened with the remaining $36-14=22$ pits? As the lowest negative pressure was not able to nucleate them, they must already have been nucleated during the first two pulses, i.e., the nucleation took place below optical resolution. This is possible as the resolution of our optical detection is limited and the pressure pulse in this case is relatively weak (i.e., $\dot{R}$ is small). Therefore, in contrast with case B, we are not able to measure the pressure for which nucleation does not take place. When the third pulse $\left(p_{m}=-0.34 \mathrm{MPa}\right)$ was applied without the other two preceding pulses, the full pattern became visible [Fig. 8(d)]. Hence, this is the pressure level for which we are sure that nucleation of the full pattern takes place.

Finally, sample D was studied, which consists of nanopits with $50 \mathrm{~nm} \leq r_{c} \leq 60 \mathrm{~nm}$ and varying depths. Just like sample $\mathrm{C}$, the shallow pits on sample D nucleated only a few times, and could not be nucleated in later experiments. Fortunately, two columns with the deepest pits $\left(d_{c}=500 \mathrm{~nm}\right.$ and $r_{c}=57 \mathrm{~nm}$, and $d_{c}=1000 \mathrm{~nm}$ and $\left.r_{c}=60 \mathrm{~nm}\right)$ could be nucleated repeatedly, and the nucleation threshold could be measured for these pits. The experiment was very similar to the ones described before, but now the sample was $12 \mathrm{~mm}$ away from the acoustic focus. A typical experimental result is depicted in Fig. 9. First, a pressure pulse with $p_{m}=-2.3 \mathrm{MPa}$ was applied and one pit from the right column $\left(r_{c}=60 \mathrm{~nm}\right)$ was nucleated. A stronger second pulse $\left(p_{m}=-2.6 \mathrm{MPa}\right)$ was able to nucleate the remaining five pits from this column although other pits in the sample did not nucleate, as they were smaller. Reducing the negative pressure further $\left(p_{m}=-3.0 \mathrm{MPa}\right)$ resulted in the nucleation of the left column of pits with $r_{c}=57 \mathrm{~nm}$. Hence, a small variation in pit sizes of just a few $\mathrm{nm}$ is reflected in a different nucle- 


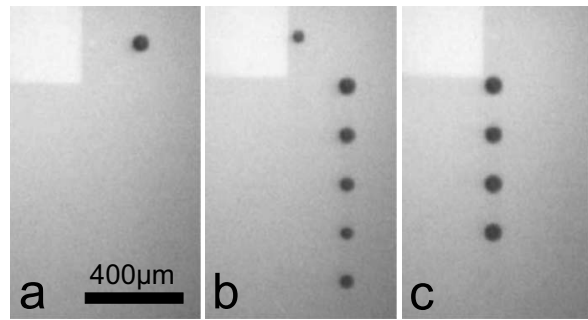

FIG. 9. Cavitation bubbles emerging from $2 \times 6$ cylindrical pits (sample D) with $r_{c}=57 \mathrm{~nm}, d_{c}=500 \mathrm{~nm}$ (left column) and $r_{c}=60 \mathrm{~nm}, d_{c}=1000 \mathrm{~nm}$ (right column), for three successively applied pressure pulses: (a) $p_{m}=-2.3 \mathrm{MPa}$; (b) $p_{m}=-2.6 \mathrm{MPa}$; (c) $p_{m}=-3.0 \mathrm{MPa}$.

ation threshold. It is also observed that the pits did not nucleate a second time, despite their huge aspect ratios.

\section{Comparison with theoretical prediction}

How do the experimental results compare with theoretical predictions? In Fig. 10 the theoretical nucleation threshold (line), based on Eq. (2), is plotted as a function of the pit radius $r_{c}$ together with the experimental results (symbols). We used $d_{c}=2 r_{c}$ as is the case in samples A and B. Note that for the pits present in sample $\mathrm{D}$ the gas pressure term is negligible: The difference between $d_{c}=2 r_{c}$ and $d_{c}=20 r_{c}$ changes the theoretical prediction for pits of $r_{c}=50 \mathrm{~nm}$ $<1 \%$. The experimental data points at which nucleation of the full pattern was detected for samples A, B, and D are depicted by crosses, while the experimental pressures where nucleation of the full pattern (just) did not happen are marked with circles. The inset shows the experimental results for sample D including typical error bars depicting the standard deviation of the pressure recordings.

We observe a striking quantitative agreement between theory and experiment for all samples. Pressure amplitudes for which nucleation was first detected are below the line marking the nucleation threshold. Pressure amplitudes for

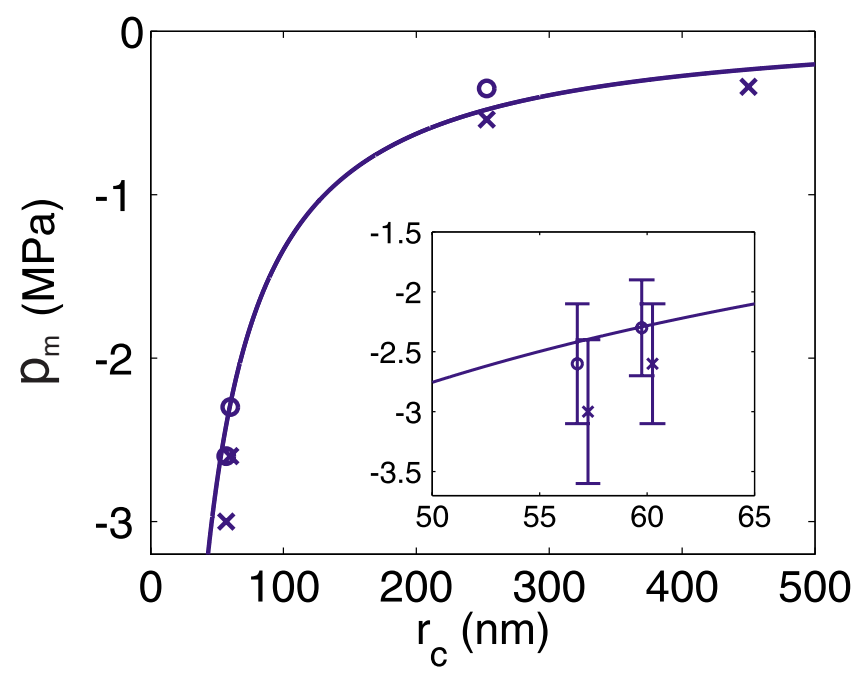

FIG. 10. (Color online) Nucleation threshold as function of the pit radius for both theory (line) and experiment (symbols, crosses: nucleation, circles: no nucleation). The inset shows a zoom in with error bars. For visibility overlapping points are shifted $\pm 0.25 \mathrm{~nm}$ with respect to each other. which nucleation did not occur are either above this line, i.e., in the regime where nucleation is not expected, or the line is within experimental error bars. For sample D it was observed that the nucleation threshold strongly depends on the size of the pits: pit radii just a few nanometer smaller resulted in a significantly lower nucleation threshold, in agreement with the steep slope of $p_{m}\left(r_{c}\right)$ around these values.

\section{Deactivation of cavitation nuclei}

It is well known that artificial nucleation sites in boiling continue to be active for a long time, emitting many bubbles. ${ }^{30}$ Similarly, the microscopic wall cracks and scratches in a glass full of beer or champagne are seen to emit bubbles for a very long time. ${ }^{31}$ Even in cavitation studies on bare hydrophobic substrates, bubbles trapped in localized defects could be nucleated more than a hundred times. ${ }^{20}$ Thus, there is something special in the deactivation of nuclei observed here which makes this situation different from the others. What is the physical mechanism responsible for the deactivation of the nuclei?

The main mechanism responsible for the deactivation of nuclei is encountered during the collapse phase of the bubble where a jet is formed. In the case of a single bubble (or weak interaction among bubbles) the jet momentum is expected to be directed to the bottom of the crevice. ${ }^{32,33}$ When bubblebubble interaction is non-negligible, on the other hand, the jet momentum may be deflected away from the crevice. ${ }^{22}$

In order to shed light on this proposed deactivation mechanism, numerical simulations were carried out to elucidate the shape of the air-liquid interface during the bubble collapse. For this we used the boundary-integral method described in Ref. 34 based on a potential flow description of the liquid dynamics. The liquid-solid angle was prescribed to be $\theta_{a}=124^{\circ}$, which corresponds to the experimentally determined advancing contact angle of water on the substrates. At the starting point of the simulations the bubble was assumed to be a segment of a sphere with a radius significantly larger than the cavity radius $r_{c}$, see Fig. 11(a). The pressure inside the bubble was assumed to be uniform, satisfying the adiabatic relation $p_{0} / V_{0}^{1.4}=p_{\text {bub }} / V_{\text {bub }}^{1.4}$ with the initial pressure $p_{0}$ and $V_{0}$ the volume of the crevice, and $p_{\text {bub }}$ and $V_{\text {bub }}$ the instantaneous pressure and volume of the bubble, respectively. After release the bubble begins to shrink rapidly due to both surface tension and the low internal pressure. Eventually it evolves into an almost cylindrical shape as illustrated in Fig. 11(c). This air cylinder collapses radially and finally closes in a single point on the axis of symmetry, leaving a small air bubble entrapped above the pinch-off point, see Fig. 11(d). Toward pinch-off the liquid rushing radially inward has to accelerate more and more to satisfy the requirement of mass conservation. When the advancing liquid front reaches the axis of symmetry a high pressure develops and the flow is deflected up and down to form two fast, needlelike water jets. The continuing collapse of the air cavity below and above the pinch-off point provides additional momentum to the two jets. ${ }^{35}$ The downward jet protrudes deeply into the cavity until it hits the bottom of the cavity, as illustrated in Figs. 11(e) and 11(f). For simplicity we ne- 

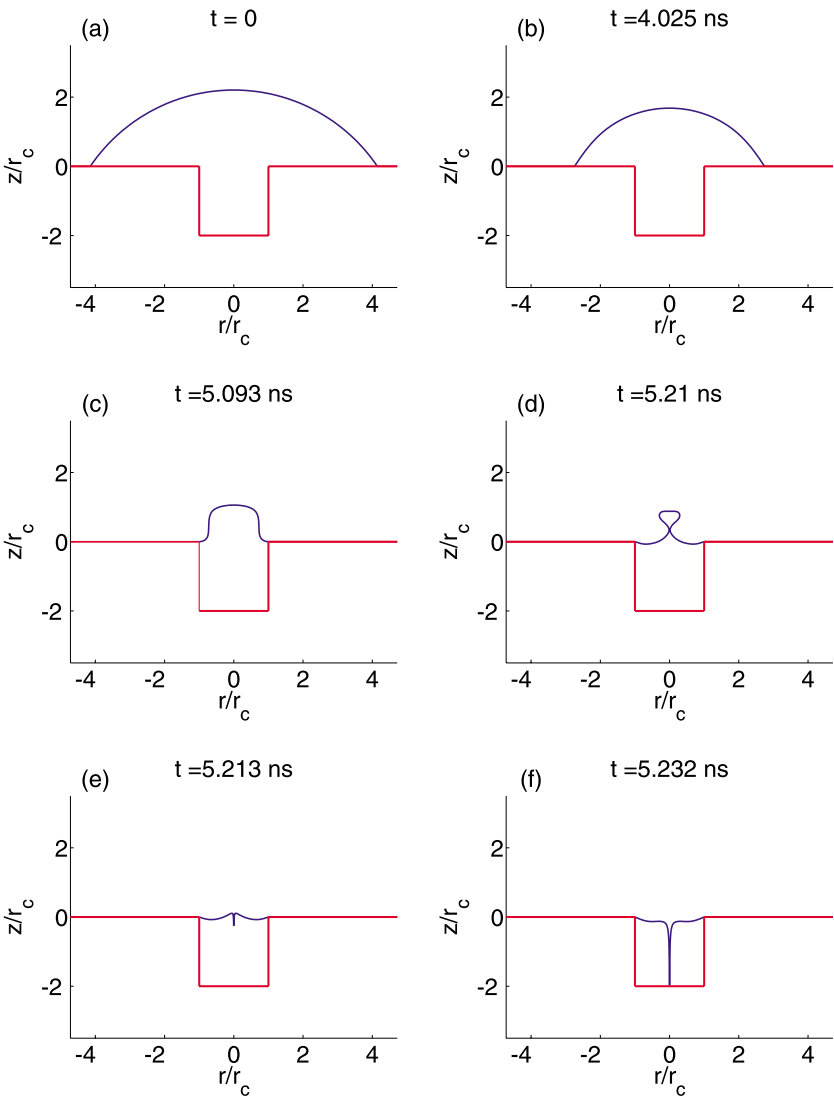

FIG. 11. (Color online) (a) Initial configuration for a spherical bubble with radius $5 r_{c}$ on top of the cavity. The (advancing) contact angle is $\theta=124^{\circ}$. Due to the low pressure inside the cavity (b) the bubble starts to collapse (c) evolving into an almost cylindrical shape, (d) which eventually closes on the axis of symmetry in a single point. (e) From the pinch-off location a downward jet protrudes into the cavity (f) eventually hitting the cavity bottom. Here it would cause a splash filling the cavity with liquid.

glected the upper bubble here which is expected to have only negligible influence on the downward jet. Upon reaching the bottom the impacting jet would form a violent, nonaxisymmetric splash which cannot be captured by our numerical technique. Nevertheless, one can easily imagine the continuation of the process: As more and more liquid enters the cavity through the jet, the cavity is flooded with liquid, making a second nucleation impossible. We checked that the jet mechanism is present for pits in the size range studied here (50 $\mathrm{nm}<r_{c}<500 \mathrm{~nm}$ ) and is independent of the initial bubble size, pit depth, and contact angle.

The previous explanation is not applicable to the case of strong mutual interaction between the bubbles, when the jet tends to be deflected away from the bottom of the crevice, ${ }^{22}$ although the nuclei are still observed to be deactivated after emission of the first bubble. We estimated that within the typical lifetime of the bubble (typically $t_{b} \sim 10 \mu \mathrm{s}$ ), the gas molecules have ample time to fill the bubble volume to reach a uniform gas pressure. The gas transport is probably a combination of convection and diffusion, although the latter mechanism alone would already be sufficient to move all the gas: The typical diffusion length scale is $\sim \sqrt{D t_{b}}=10 \mu \mathrm{m}$, using the diffusion coefficient $D \sim 10^{-5} \mathrm{~m}^{2} / \mathrm{s}$. In the cases studied here the pits are $<1 \mu \mathrm{m}$ deep, i.e., much smaller

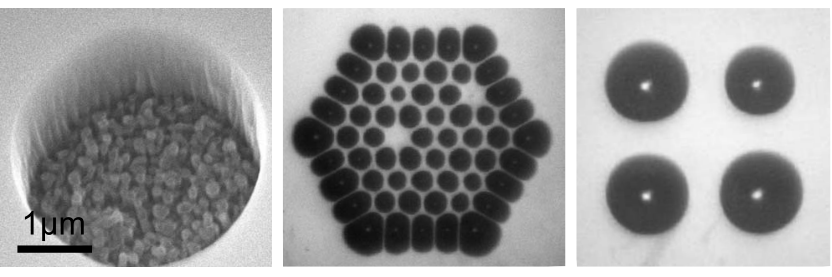

FIG. 12. A superhydrophobic pit (left) can be nucleated hundreds of times, provided that the liquid jet in the bubble collapse phase is not directed into the pit. Center: a hexagonal pattern of superhydrophobic pits $(100 \mu \mathrm{m}$ in between the pits) after 230 nucleation events show only two defects. Right: a square pattern (300 $\mu \mathrm{m}$ in between the pits) is completely intact after 100 shots.

than the typical diffusion length scale. Also, the volume of a typical bubble is $10^{5}-10^{7}$ larger than the volume of the nanopits, allowing the majority of the gas to move from the pit into the bubble. ${ }^{36}$ During collapse, the interaction between bubbles breaks the bubble in such a way that a large gas bubble goes away from the sample, and only a tiny fraction $(<0.001 \%)$ of the initial gas remains in the pit, which therefore remains essentially full of vapor and is easily filled by the liquid. Suppose that during this filling process a tiny amount of gas remains in the pit. The contact angle being larger than $90^{\circ}$ would force the meniscus to be curved toward the gas and consequently the bubble would grow by diffusion. We estimated that the waiting time in between two successive shots (in our case: $15 \mathrm{~s}$ ) would then be enough to refill the pits completely with gas by diffusion, and a second nucleation event should then be possible. Since we never have observed a second nucleation, we conclude that the pits have to be filled with liquid completely during bubble collapse.

These conclusions agree with our experimental results. In cases $\mathrm{A}-\mathrm{D}$ we observed no differences between strong (Fig. 6) and weak (Figs. 7-9) bubble interaction: In both situations the pits were emptied after one nucleation event, in line with the explanations provided here.

This situation can be compared to the previously mentioned continuous and long-lived emission of bubbles in carbonated beverages and the related phenomena observed with enhanced surfaces frequently used in boiling heat transfer. $^{37,38}$ In none of these cases the bubbles collapse and therefore the primary mechanism for the filling up and consequent deactivation of the pits is present.

\section{E. Superhydrophobic nuclei}

To show the importance of the liquid jet into the pits, experiments using superhydrophobic nuclei are illustrative. Cylindrical pits, etched in $\mathrm{Si}(100)$ with a diameter of $4 \mu \mathrm{m}$, were created with a superhydrophobic bottom layer (see Fig. 12 ), consisting of hydrophobic pillars of $\sim 100 \mathrm{~nm}$ in diameter (created through a black silicon etching process ${ }^{39}$ and a hydrophobic fluorocarbon top layer). The combination of hydrophobicity with roughness is known to create superhydrophobicity $^{40}$ with typical contact angles $>160^{\circ}$. When the bubbles were strongly interacting (leading to wallparallel jets), we observed that the pits remained active nucleation sites even after hundreds of shots (see Fig. 12). 
On the contrary, in the case of a single bubble (wall-normal jet) we observed that the micropits were deactivated after a few nucleation events. To explain this striking difference one really has to take the direction of the liquid jet into account. Apparently, the wall-parallel jet is not able to wet the superhydrophobic bottom of the pits, while the vigorous, ultrathin jet directed toward the superhydrophobic bottom layer presumably pushes the liquid from the dewetted into the wetted state. From other work it is indeed known that a force may be required to overcome the energy barrier associated with this wetting transition. ${ }^{41}$ Once in the wetted state, the superhydrophobic pit is deactivated and cannot be nucleated again, apart of course from drying the whole sample.

\section{CONCLUSION}

In conclusion, Atchley and Propseretti's 1989 crevice model of cavitation nuclei is experimentally verified using nanoscopic well-defined nuclei. Advanced etching techniques allowed us to create cylindrical pits down to $50 \mathrm{~nm}$ in radius with high accuracy in both their size, depth, and mutual position. Upon immersion in water, the hydrophobic nanopits trapped air and served as nucleation sites. Stepwise lowering of the acoustic minimum pressure allowed us to determine the nucleation threshold at which the pits start to cavitate. We found that the experimental results are in very good agreement with the theoretical predictions. This implies that in shock wave experiments the largest size of cavitation nuclei can be determined by measuring the pressure at which they start to nucleate, provided that either the cavity geometry is known so that one can incorporate the effect of the expanding gas from the pit, or that the gas content of the pit can be neglected, which is roughly the case for $r_{c}<200 \mathrm{~nm}$ (in case $d_{c}=r_{c}$ ).

Cavitation nuclei were deactivated after a single nucleation event, despite differences in width, depth, and aspect ratios of the pits. The two mechanisms contributing to this effect are diffusion of gas out of the pit during the lifetime of the bubble and the subsequent aspherical collapse of the bubble. Numerical simulations show that in the case of weak bubble-bubble interaction, a sharp wall-directed liquid jet is formed which hits the bottom of the cavities, thus vigorously wetting the pits. Superhydrophobic nuclei can only be wetted, thanks to this jet directed toward the crevice bottom. For strong bubble-bubble interactions with wall-parallel jets, superhydrophobic pits remain active nucleation sites, even after hundreds of nucleation events, in contrast to standard hydrophobic pits. In systems where one wants to control the number of cavitation nuclei which do not deactivate, superhydrophobic pits may find applications.

\section{ACKNOWLEDGMENTS}

We are grateful to Clemens Padberg for the preparation of the samples with the nanopits and Johan Bomer for the samples with the superhydrophic pits. We acknowledge Holger Schönherr for the AFM work involved. This work was supported by the NanoNed, the nanotechnology program of the Dutch Ministry of Economic Affairs (Grant No. TMM.6413).
${ }^{1}$ F. Caupin and E. Herbert, "Cavitation in water: A review," C. R. Phys. 7, 1000 (2006).

${ }^{2}$ E. Herbert, S. Balibar, and F. Caupin, "Cavitation pressure in water," Phys. Rev. E 74, 041603 (2006).

${ }^{3}$ K. A. Mørch, "Reflections on cavitation nuclei in water," Phys. Fluids 19, 072104 (2007)

${ }^{4}$ E. N. Harvey, D. K. Barnes, W. D. McElroy, A. H. Whiteley, D. C. Pease, and K. W. Cooper, "Bubble formation in animals," J. Cell. Comp. Physiol. 24, 1 (1944).

${ }^{5}$ M. Strasberg, "Onset of ultrasonic cavitation in tap water," J. Acoust. Soc. Am. 31, 163 (1959).

${ }^{6}$ R. E. Apfel, "The role of impurities in cavitation-threshold determination," J. Acoust. Soc. Am. 48, 1179 (1970).

${ }^{7}$ L. A. Crum, "Tensile strength of water," Nature (London) 278, 148 (1979).

${ }^{8}$ A. A. Atchley and A. Prosperetti, "The crevice model of bubble nucleation," J. Acoust. Soc. Am. 86, 1065 (1989).

${ }^{9}$ K. A. Mørch, "Cavitation nuclei and bubble formation-a dynamic liquidsolid interface problem," ASME J. Fluids Eng. 122, 494 (2000).

${ }^{10}$ We do not refer to "imperfectly wetted solids" as we think there is a big difference between that model and the crevice model as we understand it. The crucial aspect of the crevice model is the stabilization of gas pockets by the hydrophobic nature of the surface of the solid particle. The earlier models with imperfectly wetted solids had no gas in them. They simply postulated that the liquid was weakly adherent to the solid so that it could detach when the pressure fell. Since in this case the bubble would start with the size of the solid impurity, rather than from zero radius, surface tension would not prevent the nucleation.

${ }^{11}$ M. Greenspan and C. E. Tschiegg, "Radiation-induced acoustic cavitation: Apparatus and some results," J. Res. Natl. Bur. Stand., Sect. C 71, 299 (1967).

${ }^{12}$ L. J. Briggs, "Limiting negative pressure of water," J. Appl. Phys. 21, 721 (1950).

${ }^{13}$ R. A. Roy, S. I. Madanshetty, and R. E. Apfel, “An acoustic backscattering technique for the detection of transient cavitation produced by microsecond pulses of ultrasound," J. Acoust. Soc. Am. 87, 2451 (1990).

${ }^{14}$ S. I. Madanshetty and R. E. Apfel, "Acoustic microcavitation: Enhancement and applications," J. Acoust. Soc. Am. 90, 1508 (1991).

${ }^{15}$ S. I. Madanshetty, R. A. Roy, and R. E. Apfel, "Acoustic microcavitation: Its active and passive acoustic detection," J. Acoust. Soc. Am. 90, 1515 (1991).

${ }^{16}$ S. I. Madanshetty, "A conceptual model for acoustic microcavitation," J. Acoust. Soc. Am. 98, 2681 (1995).

${ }^{17}$ C. X. Deng, Q. Xu, R. E. Apfel, and C. K. Holland, "Inertial cavitation produced by pulsed ultrasound in controlled host media," J. Acoust. Soc. Am. 100, 1199 (1996).

${ }^{18}$ H. B. Marschall, K. A. Mørch, A. P. Keller, and M. Kjeldsen, "Cavitation inception by almost spherical solid particles in water," Phys. Fluids 15, 545 (2003)

${ }^{19}$ B. M. Borkent, M. Arora, and C. D. Ohl, "Reproducible cavitation activity in water-particle suspensions," J. Acoust. Soc. Am. 121, 1406 (2007).

${ }^{20}$ N. Bremond, M. Arora, C. D. Ohl, and D. Lohse, "Cavitation on surfaces," J. Phys.: Condens. Matter 17, S3603 (2005).

${ }^{21}$ N. Bremond, M. Arora, C. D. Ohl, and D. Lohse, "Controlled multibubble surface cavitation," Phys. Rev. Lett. 96, 224501 (2006).

${ }^{22} \mathrm{~N}$. Bremond, M. Arora, S. M. Dammer, and D. Lohse, "Interaction of cavitation bubbles on a wall," Phys. Fluids 18, 121505 (2006).

${ }^{23}$ B. M. Borkent, S. M. Dammer, H. Schönherr, G. J. Vancso, and D. Lohse, "Superstability of surface nanobubbles," Phys. Rev. Lett. 98, 204502 (2007).

${ }^{24}$ M. A. Chappell and S. J. Paine, "The effect of cavity geometry on the nucleation of bubbles from cavities," J. Acoust. Soc. Am. 121, 853 (2007).

${ }^{25}$ C. E. Brennen, Cavitation and Bubble Dynamics (Oxford University Press, New York, 1995).

${ }^{26}$ T. G. Leighton, The Acoustic Bubble (Cambridge University Press, Cambridge, 1994).

${ }^{27}$ J. Staudenraus and W. Eisenmenger, "Fiberoptic probe hydrophone for ultrasonic and shock-wave measurements in water," Ultrasonics 31, 267 (1993).

${ }^{28}$ T. M. Mayer, M. P. de Boer, N. D. Shinn, P. J. Clews, and T. A. Michalske, 
"Chemical vapor deposition of fluoroalkylsilane monolayer films for adhesion control in microelectromechanical systems," J. Vac. Sci. Technol. B 18, 2433 (2000).

${ }^{29}$ M. S. Plesset and A. Prosperetti, "Bubble dynamics and cavitation," Annu. Rev. Fluid Mech. 9, 145 (1977)

${ }^{30}$ V. K. Dhir, "Boiling heat transfer," Annu. Rev. Fluid Mech. 30, 365 (1998).

${ }^{31}$ G. Liger-Belair, G. Polidorib, and P. Jeandeta, "Recent advances in the science of champagne bubbles," Chem. Soc. Rev. 37, 2490 (2008).

${ }^{32}$ T. B. Benjamin and A. T. Ellis, "The collapse of cavitation bubbles and the pressure thereby produced against solid boundaries," Philos. Trans. R. Soc. London, Ser. A 260, 221 (1966)

${ }^{33}$ M. S. Plesset and R. B. Chapman, "Collapse of an initially spherical vapor cavity in the neighborhood of a solid boundary," J. Fluid Mech. 47, 283 (1971).

${ }^{34}$ R. Bergmann, D. van der Meer, S. Gekle, A. van der Bos, and D. Lohse, "Controlled impact of a disc on a water surface: Cavity dynamics," J. Fluid Mech. 633, 381 (2009).
${ }^{35}$ S. Gekle, J. M. Gordillo, D. van der Meer, and D. Lohse, "High-speed jet formation after solid object impact," Phys. Rev. Lett. 102, 034502 (2009).

${ }^{36}$ Notice that this estimation does not take into account the confinement of the nanopits and their large aspect ratios (case D), which may lead to lower values of the diffusion coefficient.

${ }^{37}$ A. D. Messina and E. L. Park, "Effects of precise arrays of pits on nucleate boiling," Int. J. Heat Mass Transfer 24, 141 (1981).

${ }^{38}$ Y. Qi, J. F. Klausner, and R. Mei, "Role of surface structure in heterogeneous nucleation," Int. J. Heat Mass Transfer 47, 3097 (2004).

${ }^{39}$ H. Jansen, M. de Boer, R. Legtenberg, and M. Elwenspoek, "The black silicon method: A universal method for determining the parameter setting of a fluorine-based reactive ion etcher in deep silicon trench etching with profile control," J. Micromech. Microeng. 5, 115 (1995).

${ }^{40}$ D. Quéré, "Surface chemistry-fakir droplets," Nature Mater. 1, 14 (2002).

${ }^{41}$ N. A. Patankar, "Transition between superhydrophobic states on rough surfaces," Langmuir 20, 7097 (2004). 